\title{
BIG-RIP, SUDDEN FUTURE, AND OTHER EXOTIC SINGULARITIES IN THE UNIVERSE
}

\author{
MARIUSZ P. DA̧BROWSK * and ADAM BALCERZAK \\ Institute of Physics, University of Szczecin, \\ Wielkopolska 15, 70-451 Szczecin, Poland \\ mpdabfz@sus.univ.szczecin.pl
}

\begin{abstract}
We discuss exotic singularities in the evolution of the universe motivated by the progress of observations in cosmology. Among them there are: Big-Rip (BR), Sudden Future Singularities (SFS), Generalized Sudden Future Singularities (GSFS), Finite Density Singularities (FD), type III, and type IV singularities. We relate some of these singularities with higher-order characteristics of expansion such as jerk and snap. We also discuss the behaviour of pointlike objects and classical strings on the approach to these singularities.
\end{abstract}

\section{Introduction}

Through many years in the past only the two basic cosmological type of singularities were known among the isotropic models of the universe. These were Big-Bang and Big-Crunch appended by a future asymptotic (and non-singular) state of a de-Sitter type. The appearance of Big-Bang and Big-Crunch was in no way related to any of the energy conditions violation. The progress in cosmological observations at the turn of the 21 st century ${ }^{1}$ did not add anything new to the picture apart from the fact that then it was realized that these singularities could emerge also in the strong-energy-condition-violation cases of $\varrho+3 p<0$. However, a deeper analysis of the data from supernovae, cosmic microwave background (WMAP) and largescale structure (SDSS) ${ }^{2}$ shows that there exists other possibilities of the universe evolution which admit new type of singularities and the problem of the link between energy conditions violation and the singularity appearance becomes unclear. We will discuss these new singularities and the problems to relate them with the possible generalized energy conditions as well as some new observational characteristics of the expansion of the universe.

\section{Phantom-driven Big-Rip. Phantom duality.}

The main motivation to exotic singularities comes from phantom. ${ }^{3}$ Apparently, it emerged that the observational data does not make any "borderline" at $p=-\varrho$ in cosmology and that the smaller pressure is allowed to dominate current evolution. Phantom may easily be simulated by a scalar field $\phi$ of negative kinetic energy which gives the energy-momentum tensor for a perfect fluid with the energy density $\varrho=$ $-(1 / 2) \dot{\phi}^{2}+V(\phi)$, and the pressure $p=-(1 / 2) \dot{\phi}^{2}-V(\phi)$, so that it surely violates the null energy condition since $\varrho+p=-\dot{\phi}^{2}<0$. Phantom is allowed in Brans-Dicke theory in the Einstein frame (for Brans-Dicke parameter $\omega<-3 / 2$ ), in superstring

*Presenting author. 
cosmology, in brane cosmology, in viscous cosmology and many others. The most striking consequence of phantom is that its energy density $\varrho$ grows proportionally to the scale factor $a(t)$. Then, unlike in a more intuitive standard matter case, where the growth of the energy density corresponds to the decrease of the scale factor, here, the growth of the energy density accompanies the expansion of the Universe. This allows a new type of singularity in the universe which is called a Big-Rip. This singularity appears despite all the energy conditions are violated. It is a true singularity in the sense of geodesic incompletness apart from some range of the possible equations of state for isotropic geodesics which are complete. ${ }^{4} \mathrm{~A}$ very peculiar feature of phantom models against standard models is phantom duality. ${ }^{5}$ It is a new symmetry of the field equations which allows to map a large scale factor onto a small one and vice versa due to a change

$$
a(t) \leftrightarrow \frac{1}{a(t)} \quad \text { or } \quad w+1 \leftrightarrow-(w+1),
$$

with a consequence of replacing energy conditions violating matter onto a nonviolating one.

\section{Sudden (and Generalized) Future Singularities, Finite Density singularities, type III and IV singularities.}

Big-Rip leads to violation of all the energy conditions. It appears that one is able to get some other exotic singularities which violate the dominant energy condition $(p<|\varrho|)$ only or even do not violate any energy condition. The former are SFS and the latter are GSFS. The idea to get them is not to constrain the set of cosmological field equations by any equation of state, ${ }^{6}$ which allows an independent evolution of the energy density and the pressure. Actually, the energy density depends on at most first derivative of the scale factor, while the pressure depends on the second derivative, too. Then, it may happen that at a certain moment of the evolution only the second derivative of the scale factor is divergent - this is a Sudden Future Singularity - the energy density remains finite, while the pressure blows-up to infinity. It was shown that it is a weak singularity ${ }^{4}$ in the sense of the formal definitions of singularities known in general relativity. The main point is that there is no geodesic incompletness at this singularity and the evolution of an individual pointlike object can be extended through it. Same refers to Generalized Sudden Future Singularities. These singularities are temporal (appear at some fixed time on a hypersurface $t=$ const.), but there exist also a spatial pressure singularities (may exist somewhere in the universe nowadays) in cosmology, though in inhomogeneous models. ${ }^{7}$ It is possible to have inhomogeneous models of the universe which exhibit both types of singularities. Finally, other exotic types of singularities are also possible. ${ }^{8}$ These are type III (with finite scale factor and blowing-up the energy density and pressure) and type IV (with finite scale factor, vanishing the energy density and pressure, blowing-up the pressure derivative). It is interesting to know the difference between the evolution of pointlike objects and extended objects such as fundamental strings 
through these various exotic singularities. ${ }^{9}$ As it was mentioned already, the pointlike objects are really destroyed in a Big-Rip singularity only. However, at SFS the infinite tidal forces appear, and one may worry about the fate of strings approaching these singularities. It was shown ${ }^{9}$ that this is subtle in the sense that strings are not infinitely stretched (remain finite invariant size) at any of these singularities apart from a Big-Rip. In other words, extended objects like strings, despite infinite tidal forces, may cross through SFS, GSFS, type III, and type IV singularities.

\section{Generalized energy conditions and exotic singularities.}

From the above considerations it is clear that the application of the standard energy conditions to exotic singularities is not very useful. Then, one should try to formulate some different energy conditions which may be helpful in classifying exotic singularities. ${ }^{10}$ This may be put in the context of the higher-order characteristics of the expansion (statefinders) which involve higher-order derivatives of the scale factor such as jerk, snap etc. ${ }^{10,11}$ For example, one could think of a hybrid energy condition like $\alpha \varrho>\dot{p}$ with $\alpha=$ const., to prevent an emergence of SFS, or a higherorder dominant energy condition in the form $\dot{\varrho}>|\dot{p}|$, whose violation can be a good signal of GSFS.

\section{Conclusion}

Universe acceleration gave some motivation to study non-standard cosmological singularities such as Big-Rip, Sudden Future Singularity, Finite Density singularity and type III, IV singularities. However, most of these singularities (apart from BigRip) are weak singularities which do not exhibit geodesic incompletness and allow the evolution of both pointlike objects and strings through them.

\section{Acknowledgments}

This work has partially been supported by the Polish Ministry of Science and Education grant No 1P03B 04329 (years 2005-07).

\section{References}

1. S. Perlmutter et al., Astroph. J. 517, 565 (1999).

2. M. Tegmark et al., Phys. Rev. D 69, 103501 (2004).

3. R.R. Caldwell, Phys. Lett. B 545, 23 (2002).

4. L. Fernandez-Jambrina and R. Lazkoz, Phys. Rev. D 70, 121503(R) (2004).

5. M.P. Da̧browski, T. Stachowiak and M. Szydłowski, Phys. Rev. D 68.

6. J.D. Barrow, Class. Quantum Grav. 21, L79 (2004); ibid. 21, 5619 (2004).

7. M.P. Da̧browski, Phys. Rev. D 71, 103505 (2005).

8. S. Nojiri, S.D. Odintsov and S. Tsujikawa, Phys. Rev. D 71,063004 (2005).

9. A. Balcerzak and M.P. Dąbrowski, Phys. Rev. D73, 101301(R) (2006).

10. M.P. Dąbrowski, Phys. Lett. B625, 184 (2005).

11. U. Alam, V. Sahni, T.D. Saini, and A.A. Starobinsky, Mon. Not. R. Astron. Soc. 344, 1057 (2003). 